\title{
Gloria Maldonado
}

When it comes to outsourcing biomedical research and development (RED), Asia has been the big winner in the past decade. But in July, Pfizer launched its US\$21-million Center of Excellence in Precision Medicine (CEPM) in Chile, marking Pfizer's first RED move into Latin America. Gloria Maldonado, Executive Director of the newly inaugurated CEPM, speaks with Asher Mullard about the Chilean private-public partnership and the prospects for increased investment into biopharmaceutical R\&D in Latin America.

\section{Q What will you work on at the CEPM?}

We are going to validate new technology platforms that can be used for molecular diagnostics. Currently, these platforms are usually used more for basic research in universities. They have not yet reached a commercial level where they can be used for cancer diagnostics. What we want to do is validate these technologies, using tumour tissue from cancer patients, to improve patient care.

We are starting with the next-generation sequencing machine. We have partnered with Thermo Fisher Scientific, which has developed the Ion PGM (Personal Genome Machine) System, which we will validate by comparing it with gold-standard, US Food and Drug Administration (FDA)-approved testing platforms. Our goal is to compare the quality of the results from these different platforms.

We are starting with lung cancer, validating the Ion PGM's ability to pick up anaplastic lymphoma kinase $(A L K)$ and ROS 1 abnormalities. The current standards of care for identifying these genetic markers are fluorescence in situ hybridization (FISH) and immunohistochemistry. We'll compare these different platforms using 6,000 patient tissue samples. Because Chile is a small country, we couldn't get to that number of patient samples on our own, so we have teamed up with Genoma Mayor in Chile and with a genomics centre in Brazil to reach more patients. Together, these three genomic centres will become the largest genomic platform in Latin America. That is an enormous achievement, and we hope to expand to include other countries as well in future research projects.

We are already talking with different partners about the possibility of validating diagnostics platforms for other cancers as well. We have started a project in breast cancer. And gastric cancer is another attractive option, because we have a high incidence of gastric cancer in Latin America.
Q What are the benefits of using a next-generation sequencing platform as opposed to FISH or immunohistochemistry? One thing that is attractive about a next-generation platform like the Ion PGM is that it should provide better accuracy. It also needs less tissue. It needs only very small slices of tissue to be able to determine a positive result, so at the end of the day it could be less invasive for patients.

\section{Q Beyond validating diagnostics,} do you think this project could provide other biopharmaceutical R\&D gains? Yes. The gene-sequencing panel that we are going to use can detect mutations in 53 genes, so we are certain that we can add other value. We are already discussing several different approaches to making use of these data. We could strengthen our epidemiological know-how - for example, by studying how Latin American tumour genetics differ from those described in well-characterized populations. The data might also point to new potential targets. Another very attractive possibility would be to see if we can make new use of our portfolio of drugs to treat specific cancers that are more common in Latin America.

\section{Q. And this is Pfizer's first foray into} biopharmaceutical R\&D in Latin America? To date, all of Pfizer's research centres have been located in the Northern Hemisphere: in Canada, the United States, Europe and

This is the first time that Pfizer has done something like this in an emergent economy in the Southern Hemisphere

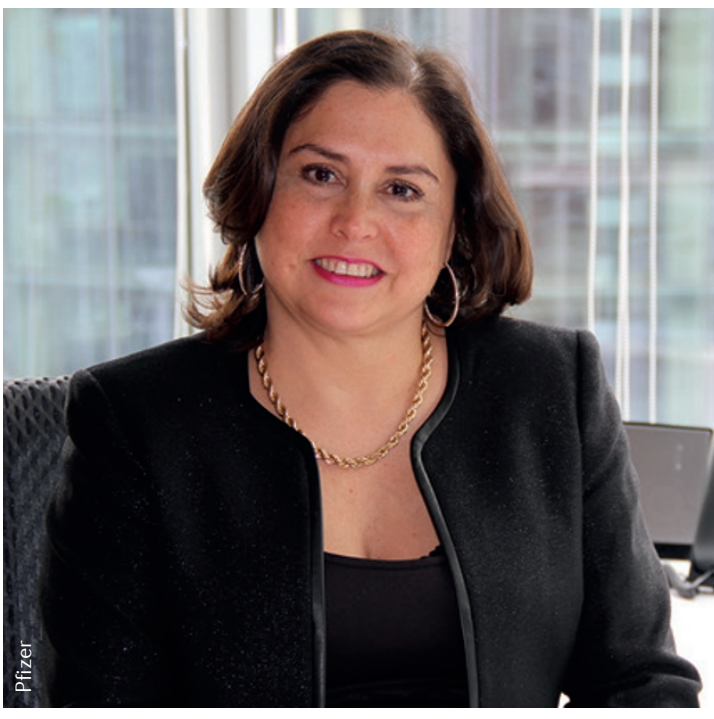

China. This is the first time that Pfizer has done something like this in an emergent economy in the Southern Hemisphere. It is also the first time that Pfizer has teamed up with a Latin American government to develop an R\&D effort. Pfizer has made a bet on a small Latin American country with a small market. We are extremely proud of this.

Q Why do you think Pfizer was interested in setting this centre up in Chile?

Chile has long been a commodity economy, and copper has been our main productive sector. But our copper is starting to be more expensive to produce, and we foresee a maximum of maybe 50 more years of production. That has created an urgent drive for the country to develop a different type of economy, and we see an R\&D focus as a critical survival strategy.

As a result, Chile has worked to promote an innovative ecosystem. It has provided lots of subsidies to everything from basic research to sophisticated, large-scale applied-research projects. It has also created an R\&D tax credit benefit that encourages companies to set up research centres in Chile. These efforts mean it made sense for Pfizer to come and set up the CEPM here. The Chilean government is also a strategic partner of the CEPM, and that relationship has been extremely helpful in terms of opening doors and developing networks.

From my perspective, Chile - and other countries like Brazil, Argentina and Colombia - really have to tap into the life sciences sector. There is so much knowledge here, and we just need the right push to be able to develop the next drug of the future. I hope that this model of private-public partnership will be replicated in other countries in Latin America. I think it is a very successful model. 